Ulrike Ravens-Sieberer

Abstract The mental health of children and adolescents is an epidemiological outcome of considerable public health relevance. This is because of a comparatively high prevalence of mental health problems and disorders in children and adolescents. The 'BELLA Study on Mental Health', a mod-

Prof. Dr. U. Ravens-Sieberer, MPH ( $ه)$ Dept. of Psychosomatics in Children and Adolescents

Centre for Obstetrics and Paediatrics

University Clinic Hamburg-Eppendorf

Building W 29 (Erikahaus)

Martinistraße 52

20246 Hamburg, Germany

Tel.: +49-40/42803-7378

Fax: +49-40/42803-5105

E-Mail: ravens-sieberer@uke.unihamburg.de

\section{The contribution of the BELLA study in filling the gap of knowledge on mental health and well-being in children and adolescents in Germany}

ular component of the Health Interview and Examination Survey for Children and Adolescents conducted by the Robert KochInstitute, assessed the prevalence of general and specific mental health problems in a representative sample of children and adolescents in Germany. Furthermore, determinants of mental health were studied in a developmental context. In the cross-sectional part of the study, participants were interviewed to assess the occurrence of risk factors and available assets (protective factors) for mental health, providing valuable data for health monitoring and planning. The sample was also repeatedly studied in a 2-year longitudinal design in order to estimate the effect size of risk and protective factors for mental health, and to analyse their combined effects on the process of mental health development. The BELLA study - as described in this supplement - supplies valuable estimates of the prevalence of mental health problems and disorders, identifies risk groups needing intervention, and describes the subjective health of children and adolescents in Germany.

Key words mental health children - health survey
New morbidity is a term frequently used in public health to signify the changing trends in disease prevalence and occurrence. Instead of acute, predominantly infectious diseases, we are now facing an era of chronic diseases, which are in most cases incurable and demand long-term treatment. While physical diseases continue to be the primary cause of morbidity, mental health disorders are also producing adverse effects and therefore demand greater attention. Reports of mental health problems in children are particularly worrisome. Overall, approximately $20-25 \%$ of the burden of disease in the European
Region is estimated to be due to mental health diseases [4]. Without representative epidemiological data, however, it is impossible to draw any conclusions and undertake suitable prevention and intervention. To do so, more reliable data is needed.

In view of this situation, a representative survey on mental health in children and adolescents, the BELLA study, was conducted in children and adolescents in Germany. The children and adolescents surveyed are a subsample drawn from the "National Health Survey for Children and Adolescents in Germany' (KiGGS), a large representative cross-sectional health interview 
and examination survey which collected data on the health status of children and adolescents $0-17$ years of age $[10,11]$. An important aspect of this survey on the health status of children and adolescents in Germany was the assessment of data on mental and subjective health in addition to data on somatic health.

While a number of studies dealing with the prevalence of mental disorders do already exist and the effects of existing risk factors on the health of children and adolescents have been demonstrated, there are to date no large-scale epidemiological studies that allow the prevalence of mental health problems in childhood and adolescence to be linked to biological, family and social risks on the one hand, or to personal, family and social resources on the other hand. Such a link is established for the first time in the BELLA module described here, in connection with the first German health survey among children and adolescents, and it provides important insights into the development and progression of mental health problems in children and adolescents.

The BELLA module 'Mental Health' uses the basic data on mental health and subjective well-being that was collected in the course of the KiGGS survey on the health status of children and adolescents, and extends these by differentiated and specific questions and additional instruments. From the start, care was taken to ensure close coordination between the module and the main survey, and to keep the burden on the participants as low as possible. For the BELLA module, a subsample of all the subjects between the ages of 7 and 17 years who had taken part in the main survey was selected so as to be representative of the overall German population. These participants were informed at the time of their appointment for the main survey about this additional survey module.

The aim of the BELLA study was to provide reliable data on the prevalence of general and specific mental health problems and to identify and examine determinants of mental health in a developmental context. The BELLA study therefore makes it possible to expand and deepen the insight related to mental health gained in the main survey. Cross-sectional data for the BELLA study was collected at baseline, and these participants were also included in a 2-year longitudinal design.

The module set out to answer the following principal questions:

- What is the prevalence of specific mental health problems in childhood and adolescence? The main survey provides screening data on the presence of symptoms of mental health problems in the children and adolescents examined. The BELLA module follows up and expands on these data by collecting additional information about the prevalence of general and specific mental health problems.

- What risks and resources can be identified in terms of the mental development of children and adolescents? How do the risks and burdens to which children and adolescents are exposed affect their mental and subjective health (quality of life), and what protective resources are available to them?

- What are the effects of risks and resources over time? How do risks and resources contribute to the rise of mental health problems? Insights gained from the findings on the interactions and effect strengths can be used to identify approaches needed for specific prevention and intervention measures.

- How are children and adolescents with mental health problems being attended to, and how does the utilisation of health care services differ between children and adolescents with treated and untreated medical pathways?

The cross-sectional component of the BELLA study collected nationwide data on mental and subjective health, in particular on the prevalence of problems such as depression, and attention deficit/hyperactivity syndrome as well as establishing the presence of specific determinants of mental health (risk factors, personal, social and familial resources) in the age group of school children and adolescents. This allows high-risk groups requiring intervention to be identified. With the help of repeated interviews at 1-year intervals conducted over a period of 2 years in the longitudinal part of the study, it will be possible to draw additional conclusions about differential pathways of the children's and adolescents' mental health development in the future.

This unique approach and the combination with the KiGGS survey made it possible to deepen the information from the main survey by employing more specific research questions and instruments. The numerous mental health outcomes and health determinants studied in the cross-sectional part of the BELLA study are presented in this supplement.

The results of the KiGGS and BELLA data also reveal a shift in the range of diseases towards chronic and mental disorders. Roughly one in five children or adolescents displays signs of mental health problems. These include anxiety in approximately ten percent of 7- to 17-year olds, conduct disorders in seven to eight percent and depression in five to six percent. Furthermore, two to three percent of children and adolescents that age displayed symptoms of attention deficit/hyperactivity disorder (ADHD) [14]. Subjective impairment due to mental health problems result in reduced quality of life. The personal, familial 
and social assets of children and adolescents are relevant as protective factors safeguarding their mental and subjective health. A troubled climate within the family and a low socio-economic status are particularly noticeable as risk factors.

In contrast to the prevalence rates, the actual utilisation rate is a great deal lower, which is why utilisation statistics do not allow deductions to be drawn about the true prevalence of mental disorders. Thus - based on the results of the BELLA study - only approximately $25 \%$ of the parents of affected children and adolescents make use of the offer of counselling or treatment indicating that mental health problems may be inadequately attended to [15].

We do know that health care services for mental health disorders in children and adolescents are lacking - not only in Germany, but in Europe in general, since a fifth of the countries in the European Region lack programmes for children and adolescents [20].

Mental diseases are prolonged, cause high costs to the health care system and are associated with distinct disadvantages for the persons afflicted. This fact has not received adequate attention in Germany so far, so that the measurement of mental health and its treatment in childhood and adolescence in representative epidemiological studies and surveys as an aspect of public health has until now been unjustifiably neglected. In view of the fact that most mental disorders in adulthood originate in disorders of childhood and adolescence, this needs to be rectified. The stage of life at which children and adolescents find themselves must therefore receive more attention in prevention and intervention as a means of reducing follow-up costs.

Targeted measures for improving mental health in children and adolescents require well-founded information. Thanks to the BELLA study, this is now available. A mental health policy should aim to encourage the healthy mental development of children and adolescents - particularly of those at increased risk - through suitable measures and to identify and treat mental health problems at an early stage. It therefore seems wise to expand the routine, early diagnosis examinations conducted on children to include mental health aspects, and to determine whether the instruments used in the KiGGS and BELLA surveys could be used in the routine examination of children.

Against this background, the additional BELLA survey on the mental health of children and adolescents in Germany can be seen to represent an important clinical, descriptive epidemiological and health policy enhancement to the value of the entire project. The close link between the variables of the main survey and those of the module not only con- nects the spectrum of somatic disorders and mental health problems cross-sectionally, it also allows the examination of the ways in which determinants of mental health work over time. This means that future analyses will permit important insights into the development and progress of mental health in children and adolescents. The articles about the BELLA study in this supplement of European Child and Adolescent Psychiatry provide a picture of the mental health of children and adolescents in Germany. The contributions reflect important information obtained at the baseline of the study concerning a broad range of issues related to the mental health of children and adolescents. The supplement therefore addresses a variety of aspects, including prevalence rates, psychopathological problems, impairments due to mental health problems, protective and risk factors, and specific mental health problems, such as depression, and ADHD.

The opening article by Ravens-Sieberer and Kurth [13] describes the design of the study and the methods used in the BELLA study, the Mental Health Module within the German Health Interview and Examination Study (KiGGS), providing a methodological framework as a basis for the other papers in the supplement.

The next three contributions to the supplement deal with the frequency of mental health problems and compare the results of the BELLA and the KiGGS study. The prevalence rates of mental health problems are presented as a main result of the BELLA study [15], followed by a contribution by Hoelling et al. [8] on the assessment of psychopathological symptoms using the Strengths and Difficulties Questionnaire in the KiGGS study. After this, Wille et al. [18] describe the level of impairment of children and adolescents due to mental health problems.

The various specific mental health problems, such as ADHD, depression, disordered eating behaviour, and suicidal behaviour, are an important part of this supplement. ADHD is dealt with in two papers, with the KiGGS and the BELLA approach being put into perspective by Huss et al. [9] and by Döpfner et al. [5], who have applied different approaches judgement of health care professionals versus parent-based usage of ICD-10 and DSM criteria - to derive prevalence rates for attention deficit/hyperactivity disorder. Bettge et al. [2] look at the severity of depressive symptoms in children and adolescents, and Herpertz-Dahlmann et al. [7] describe patterns of disordered eating behaviour along with the associated psychopathology and the impact on the health-related quality of life of the children. Finally, Resch et al. [16] examine the frequency of selfmutilation and suicidal behaviour and its psychosocial correlates. 
Four papers analyse the psychometric properties of the questionnaires used in the BELLA study, which not only revealed methodologically satisfactory results, but also interesting findings in relation to other variables used in the study. Rothenberger et al. [17] present the results for the psychometric properties of the parent Strengths and Difficulties Questionnaire, while Erhart et al. [6] compare the properties of two different ADHD questionnaires (Conner's Scale and FBB-HKS). Barkmann et al. [1] evaluate the Center for Epidemiological Studies Depression Scale for Children (CES-DC), and Bullinger et al. [3] present the psychometric properties of the KINDL-R quality of life questionnaire.

One major topic of the BELLA study is the assessment of risks, assets and health-related quality of life in association with mental health, which is outlined in the two final papers. The paper of Wille et al. [19] deals with the risks and protective factors and their role in children's and adolescents' mental health, while the closing paper of the supplement, written by Ravens-Sieberer et al. [12], describes the health-related quality of life of German children and adolescents as an outcome of mental well-being.

The BELLA study - as described in this supplement - provides valuable estimates of the prevalence of mental health problems, identifies risk groups needing intervention, and describes the subjective health of children and adolescents in Germany. In summary, the presented cross-sectional baseline data of the large-scale, 2-year prospective BELLA study represents a comprehensive picture of children's mental well-being and mental health, especially because the BELLA sample is representative for the general population of children and adolescents in Germany. When the 2-year data set is available, its evaluation should provide us with further helpful insights into the development of mental health in children and adolescents, and its determinants.

This special issue in the European Child and Adolescent Psychiatry Journal is the first of its kind, being the first to publish internationally representative data on the mental health and well-being of German children and adolescents from the crosssectional part of the KiGGS and BELLA study. Results on the longitudinal part will follow. With this issue, the editors and the authors hope to contribute towards the discussion on mental health and well-being of children, not only in Germany but worldwide.

\begin{abstract}
Acknowledgments The BELLA study was fully funded by the CDFoundation as part of the German Science Foundation. As principal investigator of the BELLA study, I would like to thank the numerous children and parents who participated in the study for their time and engagement. The BELLA study would not have been possible without the engagement of my research team on Child Public Health, especially Susanne Bettge, Nora Wille, Michael Erhart, and Veronika Ottova. Furthermore, I also wish to thank the members of the BELLA study group who were involved in the realization of this supplement. Their time investment and dedication towards this supplement has made it an important document.
\end{abstract}

\section{References}

1. Barkmann C, Erhart M, Schulte-Markwort M, BELLA study group (2008) The German version of the Centre for Epidemiological Studies Depression Scale for Children: psychometric evaluation in a population-based survey of 7 to 17 years old children and adolescents results of the BELLA study. Eur Child Adolesc Psychiatry 17(Suppl1):116-124

2. Bettge S, Wille N, Barkmann C, Schulte-Markwort M, Ravens-Sieberer U, BELLA study group (2008) Depressive symptoms of children and adolescents in a German representative sample: results of the BELLA study. Eur Child Adolesc Psychiatry 17(Suppl1): 71-81

3. Bullinger M, Brütt AL, Erhart M, Ravens-Sieberer U, BELLA study group (2008) Psychometric properties of the KINDL-R questionnaire: results of the BELLA study. Eur Child Adolesc Psychiatry 17(Suppl1):125-132

4. Bundesministerium für Gesundheit, Familie und Jugend (BMGJF) (2008) Beirat für Psychische Gesundheit. Wien
5. Döpfner M, Breuer D, Wille N, Erhart M, Ravens-Sieberer U, BELLA study group (2008) How often do children meet ICD-10/DSM-IV criteria of attention deficit-/hyperactivity disorder and hyperkinetic disorder? Parent-based prevalence rates in a national sample - results of the BELLA study. Eur Child Adolesc Psychiatry 17(Suppl1):59-70

6. Erhart M, Döpfner M, Ravens-Sieberer U, BELLA study group (2008) Psychometric properties of two ADHD questionnaires: comparing the Conners' scale and the FBB-HKS in the general population of German children and adolescents - results of the BELLA study. Eur Child Adolesc Psychiatry 17(Suppl1):106-115

7. Herpertz-Dahlmann B, Wille N, Hölling $H$, Vloet TD, Ravens-Sieberer $U$, BELLA study group (2008) Disordered eating behaviour and attitudes, associated psychopathology and health-related quality of life: results of the BELLA study. Eur Child Adolesc Psychiatry 17(Suppl1):82-91
8. Hölling H, Kurth B-M, Rothenberger A, Becker A, Schlack R (2008) Assessing psychopathological problems of children and adolescents from 3 to 17 years in a nationwide representative sample: results of the German health interview and examination survey for children and adolescents (KiGGS). Eur Child Adolesc Psychiatry 17(Suppl1): 34-41

9. Huss M, Hölling H, Kurth B-M, Schlack $\mathrm{R}$ (2008) How often are German children and adolescents diagnosed with ADHD? Prevalence based on the judgment of health care professionals: results of the German health and examination survey (KiGGS). Eur Child Adolesc Psychiatry 17(Suppl1):52-58

10. Kurth B-M (2007) Der Kinder- und Jugendgesundheitssurvey (KiGGS): Ein Überblick über Planung, Durchführung und Ergebnisse unter Berücksichtigung von Aspekten eines Qualitätsmanagements [The German Health Interview and Examination Survey for Children and Adolescents (KiGGS): an overview 
of its planning, implementation and results taking into account aspects of quality management]. Bundesgesundheitsbl - Gesundheitsforsch Gesundheitsschutz 50:533-546

11. Kurth B-M, Bergmann K-E, Dippelhofer A, Hölling H, Kamtsiuris P, Thefeld W (2002) Die Gesundheit von Kindern und Jugendlichen in Deutschland. Was wir wissen, was wir nicht wissen, was wir wissen werden. Bundesgesundheitsbl Gesundheitsforsch - Gesundheitsschutz 45(11):852-858

12. Ravens-Sieberer U, Erhart M, Wille N, Bullinger M, BELLA study group (2008) Health-related quality of life in children and adolescents in Germany: results of the BELLA study. Eur Child Adolesc Psychiatry 17(Suppl1):148-156

13. Ravens-Sieberer U, Kurth B-M, KiGGS study group, BELLA study group (2008) The mental health module (BELLA study) within the German Health Interview and Examination Survey of Children and Adolescents (KiGGS): study design and methods. Eur Child Adolesc Psychiatry 17(Suppl1): 10-21
14. Ravens-Sieberer U, Wille N, Bettge S, Erhart M (2007) Psychische Gesundheit von Kindern und Jugendlichen in Deutschland. Ergebnisse aus der BELLA-Studie im Kinder- und Jugendgesundheitssurvey [Mental Health of children and adolescents in Germany. Results from the BELLA study within the German Health Interview and Examination Survey for Children and Adolescents (KiGGS)]. Bundesgesundheitsbl - Gesundheitsforsch Gesundheitsschutz 50:871-878

15. Ravens-Sieberer U, Wille N, Erhart M, Bettge S, Wittchen H-U, Rothenberger A, Herpertz-Dahlmann B, Resch F, Hölling $\mathrm{H}$, Bullinger $\mathrm{M}$, Barkmann $\mathrm{C}$, Schulte-Markwort $M$, Döpfner $M$, as the BELLA study group (2008) Prevalence of mental health problems among children and adolescents in Germany: results of the BELLA study within the National Health Interview and Examination Survey. Eur Child Adolesc Psychiatry 17(Suppl1):22-33

16. Resch F, Parzer P, Brunner R, BELLA study group (2008) Self-mutilation and suicidal behaviour in children and adolescents: prevalence and psychosocial correlates: results of the BELLA study. Eur Child Adolesc Psychiatry 17(Suppl1):92-98
17. Rothenberger A, Becker A, Erhart $M$, Wille N, Ravens-Sieberer U, BELLA study group (2008) Psychometric properties of the parent strengths and difficulties questionnaire in the general population of German children and adolescents: results of the BELLA study. Eur Child Adolesc Psychiatry 17(Suppl1):99-105

18. Wille N, Bettge S, Wittchen H-U, Ravens-Sieberer U, BELLA study group (2008) How impaired are children and adolescents by mental health problems? Results of the BELLA study. Eur Child Adolesc Psychiatry 17(Suppl1):42-51

19. Wille N, Bettge S, Ravens-Sieberer U, BELLA study group (2008) Risk and protective factors for children's and adolescents' mental health: results of the BELLA study. Eur Child Adolesc Psychiatry 17(Suppl1):133-147

20. WHO European Ministerial Conference on Mental Health (2004) Mental health services in Europe: the treatment gap. EUR/04/5047810/B13. Helsinki, 12-15 January 2005. http://www.euro.who.int/ document/mnh/ebrief13.pdf. Accessed 22 Oct 2008 